\title{
Anatomie in Zürich
}

\author{
Von Gian Töndury, Zollikon
}

Bis um die Mitte des 17.Jahrhunderts wurde in Zürich nichts für die wissenschaftliche Ausbildung der Ärzte getan. Junge Leute, die den Arztberuf zu ergreifen wünschten, erhielten Stipendien zum Besuch auswärtiger Universitäten; angehende Chirurgen wurden rein praktisch durch erfahrene Ärzte ausgebildet. Johannes von Muralt (1645-1733), Stadtarzt, Professor für Physik und Chorherr am Großmünster, war der erste, der den Versuch unternahm, angehende Ärzte durch Privatunterricht in der Heimat wissenschaftlich auszubilden. 1671 erteilte er erstmals auch Unterricht in Anatomie. Er ist der eigentliche Gründer des anatomischen Unterrichtes in Zürich. Die ersten Vorlesungen wurden im Gesellschaftshaus «Zum schwarzen Garten» an der Stüßihofstatt abgehalten. 1677 erlaubte die Regierung auf seine Bitte hin, die Anatomie in Zürich öffentlich auszü̈ben an Leichen von Malefikanten und von solchen Personen, die mit merkwürdigen Krankheiten behaftet waren. Doktoren und Chirurgen durften zugegen sein.

1741 wurde auf dem Areal des alten Spitals bei der Predigerkirche ein besonderes Gebäude für die Anatomie errichtet, das einen Vortragssaal, einen Sektionsraum und eine kleine Stube für den Demonstrator enthielt. Es diente der Belehrung in beschreibender Anatomie, aber auch der pathologischen Anatomie und der Gerichtsmedizin.

1782 wurde auf privater Basis, unter Verzicht auf individuellen Privatunterricht, das medizinisch-chirurgische Institut gegründet, an welchem fünf akademisch ausgebildete Ärzte und drei Chirurgen unterrichteten. Es wurde 1804 zur kantonalen Anstalt erklärt und erhielt einen jährlichen staatlichen Beitrag von 600.-, später 800.- Franken. Mit der Erhebung zu einer staatlichen Anstalt gewann das Institut an Ansehen. Tüchtige Ärzte trugen dazu bei, ihm einen guten Ruf zu verschaffen, so daß die Zahl der Studenten nach und nach auf 90 und 100 stieg.

1832 wurde die Errichtung einer vollen Universität beschlossen, der auch eine medizinische Fakultät mit Klinikbetrieb angeschlossen werden sollte. Damit wurde das medizinisch-chirurgische Institut aufgehoben. Da seine Lehrer in privater Eigenschaft gewirkt hatten, verloren sie ihre Unterrichtsstunden; diejenigen, die an Spitälern angestellt waren, mußten ihre Stellen an die neugewählten Professoren abtreten. 


\section{Die Anatomie in Zürich nach Gründung der Universität 1833}

Als erster Lehrer der Anatomie an der neueröffneten Universität wirkte vom Sommersemester 1833 bis Ende 1834 Hermann Demme. Ihm folgte Friedrich Arnold, der gleichzeitig Anatomie, pathologische Anatomie und Physiologie lehrte und beauftragt wurde, zusammen mit Lukas Schönlein, Professor für spezielle Pathologie, Therapie und medizinische Klinik, den Plan für den Bau eines neuen Anatomiegebäudes auszuarbeiten. Dieses heute noch stehende und als Anatomisches Institut verwendete Gebäude wurde gleichzeitig mit dem Kantonsspital außerhalb der Stadtmauern in Fluntern gebaut und 1840 bezogen. Für die damaligen Verhältnisse war es ein großzügiger Bau, der von vielen sogar als zu großartig empfunden wurde. So bemerkt Gerold Meyer von Knonau in seinem 1846 erschienenen Handbuch des Kantons Zürich zu den Neubauten des Kantonsspitals, daß «an diesen Bauten allein die Anatomie getadelt worden sei, die auf 106567 Franken und 16 Rappen zu stehen kam, eine Summe, die sich wesentlich reduziert hätte, wenn nicht ein Hörsaal für über 150 Hörer gebaut worden wäre, der für eine so frequentierte Universität wie Berlin passend sein möchte und abgesehen von der Größe für seinen ursprünglichen Zweck nicht einmal gebraucht werden könne, weil er in akustischer Beziehung vollkommen verfehlt sei». Tatsächlich hatte der Hörsaal eine derart schlechte Akustik, daß die Stimme des Vortragenden schon in der zweiten Bankreihe von den Zuhörern nur mit Mühe verstanden wurde. Man verlegte ihn in den als Präpariersaal vorgesehenen Raum und den Präpariersaal in den unbrauchbaren Hörsaal. Schlechte Beleuchtung und Ventilation und ungenügende Heizung erschwerten aber die Arbeit der Studenten in nicht geringem Maße.

Jacob Henle (1809-1885), der Nachfolger von Friedrich Arnold, der Zürich Ende des Wintersemesters 1839/40 verließ, übernahm das neue Institut und konnte bei der Einrichtung selber Hand anlegen, so daß alles nach seinen Wünschen war; aber bereits er klagte über Raummangel. Dies ist verständlich, da die ersten Professoren neben Anatomie gleichzeitig in mehreren anderen Fächern wie vergleichende und pathologische Anatomie und Physiologie tätig waren. Das Institut enthielt neben den Kursräumen einen großen Sammlungsraum, ein Zimmer für klinische Sektionen, drei kleine Direktionsräume und einen Raum für den Prosektor.

Auf Henle, der Zürich bereits 1844 wieder verließ, folgte Joseph Engel, der 1849 durch Carl Ludwig ersetzt wurde. Bis 1852 hielt Ludwig im Wintersemester die Anatomie- und im Sommersemester die Physiologievor- 
lesung; dann übernahm Hermann von Meyer, der seit 1844 Prosektor und Privatdozent war, den Anatomieunterricht. Beim Weggang von Ludwig 1855 wurden Anatomie und Physiologie endgültig getrennt und je ein Professor für jedes Fach gewählt. Aber der Professor für Anatomie hatte auch über pathologische Anatomie zu dozieren. Erst 1862 wurde diese einem besonderen Dozenten, Eduard Kalbfleisch, übertragen. Erst von diesem Datum an existiert an der Universität Zürich die «Anatomie» als selbständiges Fach.

Von 1856 bis 1889 wirkten am anatomischen Institut zwei gleichgestellte Ordinarii: Hermann von Meyer, der gleichzeitig Direktor des Institutes war, und Heinrich Frey. Dieser hielt Vorlesungen über Histologie, vergleichende Anatomie, allgemeine Pathologie und Anthropologie; 1855 übernahm er als Nachfolger von Lorenz Oken auch noch die Zoologie. Jeder der beiden Dozenten verfügte im Institut nur über ein einziges Zimmer. Deshalb unterbreitete H.von Meyer - leider ohne Erfolg - mehrere Pläne zur Erweiterung des Institutes. Nach dem Rücktritt beider im Jahre 1889 wurde das Ordinariat von H.Frey aufgehoben, dafür die Stelle eines Prosektors wieder eingeführt. Mißstände im Institut, wo zwei ungefähr gleich alte Professoren in gleicher Stellung wirkten, hatten zu unhaltbaren Zuständen geführt, welche die Fakultät endgültig beseitigen wollte.

Als Nachfolger von H.von Meyer wurde Philipp Stöhr nach Zürich berufen mit dem Lehrauftrag «Anatomie, Histologie und Entwicklungsgeschichte». Stöhr hat als Histologe die mikroskopischen Kurse, die bereits 1845 von Albert Kölliker eingeführt worden waren, ausgebaut und so interessant gestaltet, daß auch Ärzte aus der Stadt und klinische Assistenten sie besuchten.

Auf Philipp Stöhr folgte 1897 Georg Ruge, ein Schüler von Ernst Haeckel und Carl Gegenbaur und charakteristischer Vertreter der phylogenetischen Epoche sowie der vergleichenden Anatomie der Wirbeltiere. Prosektor war Walther Felix, der durch Stöhr nach Zürich gekommen war. Beide Lehrer ergänzten sich: Ruge setzte das Schwergewicht auf die vergleichende Anatomie, Felix betonte die Entwicklungsgeschichte und die praktische Anwendung der Anatomie in der Topographie und im chirurgischen Operationskurs. Als Privatdozenten wirkten Anton Bühler von Davos und Hans Bluntschli von Zürich, der spätere Ordinarius für Anatomie in Frankfurt und Bern. Georg Ruge starb im Januar 1919; sein Nachfolger wurde Walther Felix, den ich als Student erlebt habe und der mir in lebhafter Erinnerung geblieben ist. 


\section{Die Anatomie in Zürich seit 1920}

Walther Felix hatte sich wissenschaftlich besonders intensiv und erfolgreich mit Fragen der Entwicklung des Urogenitalsystems beim Menschen und bei Wirbeltieren befaßt und den Nachweis der doppelten Anlage der Nachnieren erbracht. Zu Ferdinand Sauerbruchs Werk «Die Chirurgie der Brustorgane» lieferte er den meisterhaft klar dargestellten anatomischen Teil, zu welchem Sauerbruch im Vorwort bemerkt: «Seine gründlichen Forschungen zeitigten zum Teil völlig neue Ergebnisse und werden in bester Weise durch die schönen Bilder erläutert, die er selbst entwarf.» Zu allen Publikationen lieferte er die Illustrationen, deren Originale zum großen Teil noch erhalten sind. Sein Zeichnungstalent kam auch seinem Unterricht zugute. Seine klar aufgebauten, lebendigen Vorlesungen waren eigentlich ein Kommentar zu den Figuren, die er mit großem Schwung an die Wandtafel zeichnete. Im Zeichnen minderbegabte Studenten hatten einige Mühe mitzuzeichnen und gleichzeitig seinen Worten zu folgen. Felix beherrschte die Sprache spielend und verstand, die trockene Materie durch Einflechten von Beispielen praktischer Anwendung der anatomischen Kenntnisse im Handeln des Arztes zu beleben. Hiezu befähigten ihn ganz besonders sein reges Interesse für chirurgische Fragen, die Zusammenarbeit mit Sauerbruch und die $2 \frac{1}{2}$ jährige Arbeit als Chefarzt in einem Lazarett während des ersten Weltkrieges. Als sehr genauer und gewissenhafter Wissenschafter erwartete Felix auch von den Studenten exakte und saubere Arbeit im Präparierkurs und in den Mikroskopierkursen. Jeden Nachmittag erschien er um punkt drei Uhr im Präpariersaal, begab sich zu einer Gruppe im Kurs für Vorgerückte und demonstrierte. Er blieb den ganzen Nachmittag bei der gleichen Gruppe und verschwand dann wieder aus dem Saal. Der Tisch, an dem er demonstrierte, war rasch von vielen Studenten anderer Gruppen umzingelt, die sich die Erklärungen des Chefs nicht entgehen lassen wollten. Der persönliche Kontakt mit dem einzelnen Studenten war aber minimal, so daß es nur ganz selten vorkam, daß er bei seinem Erscheinen im Saal angesprochen und etwa um eine Erklärung gebeten wurde.

Als Prosektoren amteten Fräulein Prof. Hedwig Frey und Privatdozent Paul Vonwiller, deren Arbeit im Präpariersaal von zwei Assistenten und einigen älteren Studenten unterstützt wurde.

Das Studium war in den zwanziger Jahren viel freier, der Student selbständiger als heute. Er war auf sich selbst angewiesen. Es existierten keine Kontrollen; jeder konnte seine Arbeit nach Wunsch einteilen. Die 
offizielle Arbeitszeit im Präpariersaal war täglich auf nachmittags 14-17 Uhr angesetzt. Der Student konnte aber, wenn er kein Gewicht auf die Anwesenheit von Assistenten legte, auch außerhalb dieser Zeit arbeiten. Diese Freizügigkeit war nur möglich, weil in kleinen Gruppen gearbeitet wurde und für die 100-200 Studenten genügend Leichen zur Verfügung standen. Ein Vergleich mit den Verhältnissen im letzten Jahrhundert zeigt, daß es bis 1862 keine Studienpläne gab; es herrschte größte Freizügigkeit. Voraussetzung für die Immatrikulation war für Einheimische die Maturität; für Ausländer genügte ein Leumundszeugnis.

Theodor Billroth, der 1860 auf den Lehrstuhl für Chirurgie nach Zürich berufen wurde, war gegen zu große Lernfreiheit speziell im Medizinstudium. Er fand, daß die Medizinstudenten bei dem System in Zürich mit Vorlesungen und Kursen überlastet seien und deshalb die Übersicht verlören. Billroth forderte die Fakultät auf, Studienpläne zu entwerfen, die den Studenten als Wegweiser dienen sollten, und machte besonders auf die Wichtigkeit der gründlichen Kenntnis der propädeutischen Fächer aufmerksam. «Fehlen hier die Grundlagen in den Naturwissenschaften und der Anatomie, so wird die Physiologie nicht verstanden, fehlt es in der Kenntnis vom normalen Bau und der normalen Funktion des Körpers, so werden auch die krankhaften Zustände nicht verstanden.» Er hat auch vorgeschlagen, die Vorlesungen und Kurse zu bezeichnen, die als obligatorische Veranstaltungen zu besuchen sind, um am Ende des Studiums zum Examen zugelassen zu werden. Seine Vorschläge wurden von der Fakultät angenommen; das Studium wurde in das vorklinische und das klinische unterteilt. In den beiden ersten Semestern sollten die Naturwissenschaften, im 3., 4. und 5. Semester die Anatomie mit den Präparierübungen und die Physiologie besucht werden. Die folgenden 6 Semester waren der Klinik reserviert, eine Einteilung des Medizinstudiums, die bis heute ziemlich unverändert beibehalten wurde.

Bis 1889 hatte der Studienplan, welcher den Studierenden der Medizin den Weg wies, den Charakter eines Ratgebers. Die Freiheit des Studiums und die Möglichkeit, sich einzelnen besonderen Bedürfnissen anzupassen, sollte dadurch in keiner Weise angetastet werden. Die Einführung der propädeutischen Examina im Verlaufe der 1880iger Jahre brachte eine Eindämmung der Freizügigkeit und bedingte eine Neuorganisation des vorklinischen Unterrichtes. Verlangt wurden neben bestimmten Vorlesungen zwei Semester Präparierübungen und ein Semester Mikroskopieren. Das zweite propädeutische Examen wurde ausgebaut, indem die Prüfung 
in einem praktischen und einem mündlichen Teil abgenommen wurde. In den zwanziger Jahren wurde das zweite propädeutische Examen in Zürich meistens nach 4 Semestern abgelegt. Das hatte zur Folge, daß der Präparierkurs für Winteranfänger - die Kurse fanden nur im Winter statt schon im ersten Studiensemester besucht werden mußte. Die meisten Studenten kamen frisch von der Mittelschule und hatten keinerlei anatomische Vorstellungen. Es stand ihnen die «Anleitung zu den Präparierübungen» von Ruge-Felix zur Verfügung. Das Buch war aber zu umfangreich und verlangte vom Studenten einen allzu großen Zeiteinsatz. Neben den Vorlesungen in den naturwissenschaftlichen Fächern und den Vorlesungen in systematischer Anatomie fand ein Student, der noch andere Interessen befriedigen wollte, nicht genügend Muße für die intensive Vorbereitung auf den Präparierkurs. Dies wurde offenbar auch von den vorklinischen Dozenten empfunden. Aus diesem Grunde wurde über die Reorganisation des Studiums diskutiert und schließlich ein neues Examensreglement verabschiedet und in Kraft gesetzt, das die Studiendauer von 11 auf 13 Semester erhöhte. Von den 5 vorklinischen Semestern waren die beiden ersten ausschließlich den Naturwissenschaften vorbehalten.

Walther Felix starb am 17. März 1930 und wurde durch Walther Vogt ersetzt, der auf das Wintersemester 1930/31 von München, wo er Leiter der Abteilung für Histologie und Embryologie war, nach Zürich kam. Vogts kurzes, nur $4 \frac{1}{2}$ Jahre dauerndes Wirken in Zürich brachte zwar neue Gedanken und frische Luft ins Institut, war aber zu kurz, um Spuren zu hinterlassen. Der Unterrichtsplan blieb unverändert. In den formell und didaktisch hervorragenden Vorlesungen wurden funktionelle Überlegungen in den Vordergrund gestellt, so daß die Anatomie wirklich zu einer Lehre des Baues des lebenden Menschen wurde. Seine großen Fähigkeiten, seine liebenswerten menschlichen Eigenschaften und der hohe Rang seiner wissenschaftlichen Arbeiten hatten ihm schnell das Vertrauen der Studenten und engeren Mitarbeiter und die Achtung der Kollegen gesichert. Der Unterricht und das Institut nahmen aber Vogt leider so völlig in Anspruch, daß er seine wissenschaftlichen Arbeiten in Zürich nicht so weiterführen konnte, wie er geplant und seine Mitarbeiter es gewünscht hätten. In Kolloquien und Vorlesungen führte er uns in die Probleme und die Technik der Entwicklungsmechanik der Organismen ein und eröffnete uns damit den Zugang zu einer Forschungsrichtung, die für Zürich neu war und für mich als seinen Assistenten den Grundstein zu meiner besonderen Vorliebe zur Embryologie legte. 
Jedem entwicklungsmechanisch interessierten Biologen ist die Vogtsche Methode der örtlichen Vitalmarkierung der jungen Amphibiengastrula mit farbdurchtränkten Agarstückchen bekannt. Diese ermöglichte ihm, ohne den Keim zu schädigen, das Entwicklungsschicksal der einzelnen Bezirke der jungen Gastrula zu verfolgen. Sie gibt auch Auskunft über die Gestaltungsfaktoren, welche die Gastrulation beherrschen. Keiner, der irgendwie an Fragen der Frühentwicklung interessiert ist, kann an dieser ebenso umsichtigen wie sorgfältigen Arbeit vorübergehen. Sie ergab die Rückprojektion der präsumptiven Organanlagen auf Keimbezirke der jungen Gastrula durch direktes Verfolgen der Materialverwendung in vollendeter Weise. Vogt konnte mit seiner Methode das lebendige Geschehen eines Entwicklungsvorganges so demonstrieren, daß er gewissermaßen vor aller Augen ablief. In Zürich entstanden neben Schülerarbeiten, Vorträgen und Aufsätzen die Vorarbeiten zu Lehrfilmen über die Furchung, Gastrulation und Neurulation des Amphibieneies.

Von den beiden Prosektoren, die Vogt in Zürich vorfand, ließ sich Privatdozent P.Vonwiller 1931 beurlauben, um einer Einladung an das Institut von Frau Prof. Lina Stern nach Moskau Folge leisten zu können. Seine Stelle wurde mit Kurt Goerttler, einem Schüler von Vogt, besetzt, der aber bereits auf das Sommersemester 1934 einer Berufung nach Hamburg folgte. Mit der Vertretung der durch seinen Rücktritt erledigten Prosektur wurde der Verfasser dieses Artikels betraut.

Im Sommersemester 1935 trat Vogt die Nachfolge von Siegfried Mollier in München an. Es war ihm aber nicht vergönnt, seine wissenschaftliche Arbeit zu vollenden. Eine schwere offene Kniegelenksverletzung, die er sich bei einem Sturz beim Skilaufen zugezogen hatte, fesselte ihn lange Zeit ans Bett. Als er wiederhergestellt war, meldeten sich die ersten Anzeichen der Krankheit, an welcher er erst 53jährig am 17. März 1941 starb.

Nachfolger von W. Vogt wurde Wilhelm von Möllendorff, ordentlicher Professor an der Universität Freiburg i. Br. Die beiden Männer, die sich auf dem Lehrstuhl der Anatomie folgten, waren von grundverschiedenem Charakter. Vogt war wortkarg, introvertiert und erschien dem, der ihn nicht näher kannte, kühl und unnahbar, ja unter Umständen konnte sich dieser verletzt fühlen, wenn Vogt anscheinend in kühler Ruhe durch ihn hindurchsah. W. von Möllendorff war ein extravertierter Mensch von gewinnender Liebenswürdigkeit und weltmännischer Elastizität. Diese Charaktereigenschaften schufen ihm sofort den Zugang zum Nächsten. Rasch besaß er die Liebe und Anhänglichkeit seiner Studenten und Mitarbeiter, so daß sich 
schon wenige Wochen nach seinem Amtsantritt ein Vertrauensverhältnis gebildet hatte, das den Übergang von der Ära Vogt in die Ära Möllendorff wesentlich erleichterte.

Als von Möllendorff nach Zürich kam, war er eine in der ganzen medizinischen Welt bekannte und hochangesehene Persönlichkeit. Er war Herausgeber der Zeitschrift für Zellforschung und mikroskopische Anatomie und Gründer und Herausgeber des Handbuches der mikroskopischen Anatomie des Menschen. Außerdem besorgte er seit 1921 die Neuauflagen des klassischen Lehrbuches der Histologie von Philipp Stöhr, das sich mit der 25. Auflage (1943) über eine Bearbeitung erhoben hat und das Gepräge eines selbständigen Werkes des Herausgebers annahm. Es bekundet den Wandel von der statischen zur dynamischen Betrachtungsweise in der Histologie.

W. von Möllendorff war kein Morphologe im engeren und üblichen Sinne. Er war führender Vertreter der funktionellen Feinbaulehre, deren Probleme ihn frühzeitig Grenzgebiete seines Faches betreten ließen. Sein Interesse wandte sich den modernen Methoden der Vitalfärbung, Gewebezüchtung und Mikrokinematographie zu. In den Zürcher Jahren stand das Zellteilungsgeschehen im Vordergrund seiner Forschung. W. von Möllendorff baute eine Methode aus, die es ihm ermöglichte, unter Zuhilfenahme des Zeitrafferfilmes den Zellteilungsmechanismus bis in seine Einzelheiten zu analysieren. Als Untersuchungsmaterial dienten Fibroblastenkulturen. Zeitmessungen ergaben, daß bei unverzögerten Normalmitosen die Anaphase etwa in der Mitte der Ablaufszeit liegt und eine Verzögerung des Gesamtablaufes der Zellteilung meist durch eine Störung in der Metaphase bedingt ist. Quellende Salze wirken in spezifischer Weise auf die einzelnen Phasen hemmend oder fördernd ein, wobei die genaue Analyse fast für jede verwendete Substanz verschiedene Eigenschaften aufdeckte.

In den letzten Jahren beschäftigte er sich mit der Wirkung biochemisch hochaktiver Substanzen wie Steroiden und kanzerogenen Kohlenwasserstoffen auf die Mitose. Mitten aus dieser Arbeit wurde er ganz unerwartet durch den Tod abberufen, so daß diese unvollendet blieb und wegen des Aufkommens neuerer und besserer Methoden, insbesondere der Elektronenmikroskopie, nicht weitergeführt wurde.

Seine funktionelle Betrachtungsweise kam auch im Unterricht zum Ausdruck. Dabei war er sichtlich bemüht, in Vorlesungen und Kursen die Kluft zwischen den Dimensionen der makroskopischen Anatomie und der Histologie zu überbrücken. Aus diesem Grunde wurde der histologische 
Kurs in seiner alten Form aufgehoben und mit den Vorlesungen koordiniert. In diesen sogenannten Mikro-Makrokursen hatten die Studenten Gelegenheit, das in den vorausgegangenen Vorlesungen Besprochene aus eigener Anschauung kennenzulernen. In kleinen Gruppen konnten sie zum Beispiel das Auge präparieren und seine baulichen Einrichtungen makroskopisch untersuchen, um dann an Hand von mikroskopischen Schnittpräparaten den Feinbau der Augenhäute und der Linse zu analysieren. Die Arbeit im Präpariersaal wurde intensiviert und durch Einführen von Testaten, in welchen sich die Studenten über ihre Kenntnisse ausweisen mußten, besser kontrolliert. Jeden Nachmittag war von Möllendorff von 14-17 Uhr im Präpariersaal anwesend, was von den Studenten natürlich sehr geschätzt wurde und die Zusammenarbeit förderte.

Ein großes Anliegen war ihm die Renovation und Erweiterung des Institutes. Bereits Jacob Henle klagte über Raummangel und Mißstände am ganz frisch bezogenen Institut. Diese veranlaßten H. von Meyer, Pläne für eine Erweiterung auszuarbeiten und diese der Erziehungsdirektion einzureichen. Er hatte aber damit keinen Erfolg; seine Pläne wurden nicht ausgeführt. Aber auch Philipp Stöhr und Georg Ruge legten Pläne vor, bis endlich 1901 ein Flügel angebaut wurde, in dem die Kurssäle, die Direktionsräume, die Anthropologie und Teile der Gerichtsmedizin untergebracht wurden. 1919 entstand ein neuer Hörsaal an der Stelle des ehemaligen Präpariersaales. Bei den Berufungsverhandlungen mit Vogt wurde ihm der Ausbau des Institutes versprochen. Pläne wurden vorbereitet; da der Kanton aber kein Geld hatte und Vogt bereits nach vier Jahren den Ruf nach München annahm, wurde der Ausbau nochmals hinausgeschoben. W. von Möllendorff erhielt dann den offiziellen Auftrag, Renovations- und Ausbaupläne auszuarbeiten, da die Verhältnisse im Haus wegen des schlechten baulichen Zustandes und der mangelhaften hygienischen Einrichtungen langsam unhaltbar geworden waren. Ende 1943, kurz vor von Möllendorffs Tod, wurde das Projekt der Erziehungsdirektion eingereicht und vom Regierungsrat genehmigt. Im Kantonsrat wurde es einstimmig angenommen und von allen Parteien unterstützt. Die auf den September 1944 angesetzte Volksabstimmung fiel aber negativ aus; der Kredit von 3,2 Millionen Franken wurde vom Volke mit großem Mehr verworfen. Das Volk verlangte mehr Krankenbetten, d.h. den Bau eines neuen Kantonsspitals und nicht Sektionsräume. Der negative Ausgang der Volksabstimmung wurde durch eine von den Krankenkassen im letzten Moment durchgeführte, perfide Kampagne herbeigeführt. Die Hoffnung auf eine Modernisierung und 
Erweiterung des Institutes mußte einmal mehr ad acta gelegt werden. Als Prosektoren arbeiteten Frl. Prof. Hedwig Frey, Wolfgang Bargmann, der mit Möllendorff von Freiburg nach Zürich gekommen war, und der Verfasser dieses Artikels. Fräulein Frey starb Ende 1938. Bargmann verließ das Institut im Herbst 1938 und übernahm eine Stelle in Leipzig. An seine Stelle trat Otto Bucher, seit 1950 Professor der Histologie in Lausanne.

Die Tätigkeit von W.von Möllendorff war überschattet von der Kriegsdrohung und dem Ausbruch des Zweiten Weltkrieges. In den letzten Jahren fühlte er, daß sein Denken und Fühlen nicht mehr mit dem seiner Umgebung harmonisieren konnte, und so kam es zu einer immer stärker fühlbaren Vereinsamung, unter welcher der gesellige Mann stark gelitten haben muß. Am 10. Februar 1944 erlag er einem Herzinfarkt. Erfüllt von Zukunftsplänen für die Forschung und den Ausbau des Institutes, ist er mitten aus seiner Arbeit jäh herausgerissen worden.

Als W. von Möllendorff starb, standen wir noch mitten im Krieg, und es war nicht vorauszusehen, wie lange dieser noch dauern werde. Für das Sommersemester 1944 wurde mir die interimistische Leitung des Institutes übertragen; zusammen mit $\mathrm{O}$. Bucher wurde ich auch mit den Vorlesungen und Kursen betraut.

Auf das Wintersemester 1944/45 erfolgte meine Wahl zum Nachfolger von von Möllendorff. Damit begann eine sehr arbeitsintensive, verantwortungsvolle, aber auch schöne und befriedigende Tätigkeit, die bis Ende des Sommersemesters 1977 währte. Ich habe bei Felix studiert und kam in meinem letzten klinischen Semester im Winter 1930/31 als Halbtagsassistent an das Institut, dessen Leitung von W. Vogt frisch übernommen worden war. 1935 habilitierte ich mich für das Gesamtgebiet der Anatomie und übernahm die Stelle des zweiten Prosektors, nach dem Tode von Frl. srof. Frey diejenige des ersten Prosektors. Meine Assistentenzeit fiel in die dreißiger Jahre, die im Zeichen einer schweren wirtschaftlichen Rezession und Arbeitslosigkeit standen. Mittel zur Förderung des akademischen Nachwuchses fehlten; ein Aufenthalt in einem ausländischen Institut, wie er heute zur Selbstverständlichkeit geworden ist, war damals ausgeschlossen.

Nach dem Debakel der Volksabstimmung über den Kredit für den Ausbau des Institutes beschloß der Regierungsrat nach genauer Inspektion des Hauses, die dringend notwendigen und nicht länger aufschiebbaren Verbesserungen durchzuführen. Diese erstreckten sich über Jahre, bis schließlich 1965 aus dem alten, aber großzügig gebauten Haus ein modernes 
Institut wurde, das, abgesehen vom chronisch gebliebenen Raummangel, allen Anforderungen der modernen Forschung genügt.

1947 wurde eine Kommission ins Leben gerufen, die sich erneut mit Fragen der Studienreform zu befassen hatte, da die Studienordnung von 1935 nicht mehr befriedigte. Die Frage einer grundlegenden Studienreform beschäftigte damals die medizinischen Fakultäten der ganzen Welt; das Bestreben nach einer besseren Anpassung des Studiums an die Bedürfnisse der modernen Medizin lag sozusagen in der Luft. Das ist nicht verwunderlich, wenn man an die außerordentlichen Fortschritte der medizinischen Kenntnisse denkt und an die damit verbundenen größeren Anforderungen, welche an den Arzt gestellt werden mußten. Hand in Hand mit diesen Fortschritten ging die Spezialisierung. Der einzelne konnte das Ganze immer weniger überblicken, so daß sich die Frage ganz von selbst stellte, ob der Studiengang noch genüge oder grundlegend geändert werden müsse. Die Arbeiten der Kommission kamen nicht so recht weiter. Aus diesem Grunde entschlossen sich die Vorkliniker, im Rahmen der gegebenen Möglichkeiten das anatomisch-physiologische Unterrichtssystem zu ändern, um es den neuen Anforderungen besser anzupassen und die ewigen Querelen wegen der Stundenzahlen, welche die Fachvertreter für ihren Unterricht beanspruchten, zu beseitigen.

1855 waren in Zürich die beiden Grundfächer des propädeutischen Unterrichtes, Anatomie und Physiologie, die bis dahin von einem einzigen Dozenten vertreten wurden, endgültig getrennt worden. Die beiden Fächer, die nach ihrer geschichtlichen Entwicklung eng zusammengehören, entfremdeten sich in den auf die Trennung folgenden Jahren mehr und mehr. Die Physiologie machte eine ganz außerordentliche Entwicklung durch, während die Anatomie Gefahr lief, zu einer Lehre von der menschlichen Leiche herabzusinken. Es brauchte einen genialen Forscher vom Format eines Hermann Braus (1868-1924), Professor der Anatomie in Heidelberg und Würzburg, um die Reichweite des anatomischen Gedankens neu zu erkennen und zu umschreiben. Nicht zuletzt unter dem Einfluß der Ergebnisse der Entwicklungsmechanik wendete man sich vom rein Deskriptiven funktionellen Fragen zu und gab dieser mehr dynamischen Betrachtungsweise auch im Unterricht der Medizinstudenten Raum. Damit war eine Wiederannäherung von Anatomie und Physiologie möglich. 1956 wurde in Zürich der Unterricht in Anatomie und Physiologie wieder vereinigt. Unter Wahrung der Besonderheiten der beiden Disziplinen wurde erstmals im Wintersemester 1955/56 an Stelle der systematischen Anatomie eine Vorle- 
sung «Anatomie, Physiologie und Biochemie der vegetativen Organe» angekündigt, an welcher sich Anatom, Physiologe und Biochemiker nach einem genau ausgearbeiteten Plan mit festgelegten Stundenzahlen beteiligten. Im darauffolgenden Sommersemester wurden in gleicher Weise das Nervensystem und die Sinnesorgane behandelt. Für die Vorlesung über den «Bewegungsapparat des Menschen» konnte ein Orthopäde, für die Topographie der Röntgenologe gewonnen werden.

Ein bedeutender Teil der Arbeit der Vorkliniker spielt sich weiter in den Kurssälen ab, da die praktische Arbeit im Präpariersaal und im physiologischen Laboratorium, die Arbeit am Mikroskop und im biochemischen Laboratorium nicht entbehrt werden kann, wenn tüchtige und zuverlässige Ärzte herangebildet werden sollen. Die Mikro-Makro-Kurse, die von Möllendorff eingeführt hatte, blieben, soweit es das zur Verfügung stehende Material erlaubte, erhalten.

«Die fortschreitende Zersplitterung der Medizin, die wachsenden Ansprüche des Spezialistentums an die Unterrichtszeit erschweren mehr und mehr den medizinsichen Unterricht» - darüber wurde bereits anfangs des 20. Jahrhunderts geklagt. Das Bestreben, alles auf ein Minimum einzuschränken, ist bis heute nicht gebannt. Die Gefahr, daß die Hochschule und insbesondere ihre medizinische Fakultät zur Fachschule wird, in welcher alles vorgeschrieben ist und dem Studenten jede Selbständigkeit und Initiative genommen werden, ist heute besonders groß.

Der Unterricht in Anatomie beansprucht sehr viel Zeit und Energie, da der Lehrstuhlinhaber in Zürich das Gesamtgebiet, d.h. die makroskopische, die mikroskopische Anatomie, die Histologie und Entwicklungsgeschichte zu vertreten hat. Die magere Dotierung mit Assistenten und die Schwierigkeit, gut ausgewiesene Leute zu finden, die sich für den Unterricht interessierten und auch gewillt waren, länger zu bleiben, wirkte sich erschwerend aus und forderte von meinen Mitarbeitern und mir einen übergroßen Arbeitseinsatz in den Präparier- und mikroskopischen Kursen. Für die wissenschaftliche Arbeit blieb besonders in den Wintersemestern nicht mehr viel Zeit übrig.

Eingeführt von Vogt, befaßte ich mich intensiv mit Fragen der embryonalen Entwicklung bei Mensch und Tier. Begonnen hat meine wissenschaftliche Tätigkeit mit einer Arbeit über die Entwicklung und Lageformen des Colon, ein Thema, das bald auch die Mißbildungen des Magen-Darmkanales beim Menschen umfaßte. Fasziniert haben mich die experimentellen Arbeiten am Molchei, für welche die embryonale Transplantation am gleichen 
oder zwischen zwei verschiedenen Keimen verwendet wurde. Die für die Experimente notwendigen Instrumente - Glasnadeln, Pipetten und feine Haarschlingen - konnten vom Experimentator selber hergestellt werden und waren so wohlfeil, daß die Kosten vom Institut getragen werden konnten, obschon wir damals über einen Jahreskredit von nur 6000 Franken verfügten.

Die Experimente an den jungen Amphibiengastrulae hatten häufig Mißbildungen zur Folge, und es war ganz natürlich, daß sich die Frage nach der spontanen Entstehung solcher Mißbildungen beim höheren Wirbeltier und beim Menschen stellte. Systematische und vergleichende Untersuchungen ergaben, daß gewisse typische kongenitale Mißbildungen, die wir beim Menschen immer wieder beobachten, einen bestimmten Bauplan haben, den wir auch bei den experimentell erzeugten gleichartigen Mißbildungen bei Triton- und Hühnerembryonen immer wieder gefunden haben. Es handelte sich darum, den Entstehungsmechanismus zu erfassen; dies war nur möglich unter genauer Beachtung der normalen, ungestörten Entwicklung.

Von besonderer Tragweite waren zwei Ereignisse: erstens die Entdekkung des australischen Ophthalmologen McAlister Gregg über die Zusammenhänge zwischen einer Schädigung des Kindes und einer in der Frühschwangerschaft durchgemachten Rötelnerkrankung der Mutter, zweitens die Thalidomidkatastrophe. Die Kinder, über welche Gregg berichtete, zeigten ein typisches Krankheitsbild: Neben einer zentralen Linsentrübung eines oder beider Augen bestanden häufig Innenohrschwerhörigkeit oder Taubheit, Herzschädigungen und psychomotorische Störungen. Bamatter in Genf prägte für diese Schädigungen die Bezeichnung Embryopathia rubeolica und verstand darunter den Folgezustand einer Viruserkrankung in der Frühschwangerschaft. Gregg hat 1948 in einer Gastvorlesung in der hiesigen Augenklinik darüber gesprochen, und ich hatte Gelegenheit, mit ihm über das medizinisch und biologisch gleich interessante Problem zu diskutieren. Der Zufall wollte, daß im darauffolgenden Jahr eine größere Rötelnepidemie in der Schweiz herrschte. Ich verdanke dem damaligen Oberarzt der kantonalen Frauenklinik und nachmaligem Chefarzt in Winterthur, Prof. Erich Glatthaar, das erste Untersuchungsmaterial, das mir einen Einblick in das Geschehen beim Embryo vermittelte. Damals war das Virus noch unbekannt. Es ist erst 1962 in den USA aus dem Nasenschleim von Rekruten, die an Röteln erkrankt waren, isoliert worden. 1963 und in den folgenden Jahren gelang seine Isolierung auch aus Embryonen und geschädigten Organen von geborenen, mit dem Rötelnsyndrom behafteten 
Kindern. Es entwickelte sich eine intensive Zusammenarbeit mit in- und ausländischen Kliniken, die Untersuchungsmaterial lieferten. Die Untersuchungsergebnisse gewährten Einblicke in den Schädigungsmechanismus des Virus und gestatteten Rückschlüsse auf den Infektionsweg. Unterstützt wurde ich von Joseph Rickenbacher und in neuester Zeit von Gonzague Kistler und Peter Groscurth, die dem Problem auch auf elektronenmikroskopischem Weg nachgegangen sind und wichtige Hinweise auf die hohe Empfindlichkeit der Gefäßendothelien gegenüber dem Virus erhielten.

Selbstverständlich stellte sich die Frage, ob auch andere Viruserkrankungen der Mutter in der Schwangerschaft eine Gefährdung für den Embryo darstellen. Diese Frage kann nicht definitiv beantwortet werden. Es scheint aber, daß nur das Rötelnvirus eine teratogene, d.h. mißbildungserzeugende Wirkung hat. Für das Verständnis der Reaktionsweise des Embryos gegenüber Viren ist die Feststellung entscheidend, daß Vorstellungen der Pathologie des Geborenen nicht auf den Embryo übertragen werden dürfen, da dieser in den ersten 4-10 Wochen über keine Abwehrmechanismen verfügt.

1937 wurde die Medizinische Fakultät beauftragt, in einem Gutachten zur Initiative über die Zulassung der Chiropraxis als Heilmethode im Kanton Zürich Stellung zu nehmen. Unser Institut hatte dazu die morphologischen Grundlagen zu liefern. v. Möllendorff übertrug mir diese Aufgabe. Bald stellte sich heraus, daß unsere Kenntnisse der Wirbelsäule nicht weit reichten, so da $\beta$ ausgedehnte Untersuchungen über die Zwischenwirbelscheiben und die Zwischenwirbelkanäle und ihren Inhalt notwendig wurden. Da ich selber an immer wiederkehrenden Rückenbeschwerden litt, habe ich mich mit größter Energie und Ausdauer in jahrelanger Arbeit bemüht, die pränatale Entwicklung der Wirbelsäule unter besonderer Berücksichtigung der Zwischenwirbelscheiben und ihr Verhalten im Verlaufe des Lebens eingehend zu analysieren. Auf diesem Gebiete hat mich besonders Karl Theiler unterstützt, dessen erbgenetische Experimente an Kurzschwanzmäusen wesentliche Einblicke in das embryonale Geschehen vermittelten. Die Ergebnisse unserer Untersuchungen sind in einer Monographie zusammengefaßt worden, die 1958 erschien. In zahlreichen Einzelarbeiten und Vorträgen habe ich versucht, meine Befunde und meine persönlichen Erfahrungen insbesondere im Hinblick auf die so häufigen Diskopathien den Ärzten näherzubringen.

Neben den kurz charakterisierten langfristigen, parallel verlaufenden und heute noch weitergehenden Arbeiten haben wir uns im Institut auch mit 
anderen Problemen befaßt. Dazu gehören Arbeiten über die Gesichtsentwicklung mit besonderer Berücksichtigung der Entstehung von Gesichtsspalten, Arbeiten über den Segmentbau der Lungen und der Lungengefäße und Arbeiten zur Entwicklung und Morphologie des Lymphgefäßsystems und des Herzens. Hier sind besonders die grundlegenden Untersuchungen über das Lymphgefäßsystem von Stefan Kubik anzuführen.

Unsere Lehrbücher stammen zum großen Teil aus Deutschland. Während des Zweiten Weltkrieges und ganz besonders in den darauffolgenden Jahren waren diese Bücher kaum mehr zu haben, so daß ich mich entschloß, ein Lehrbuch der angewandten und topographischen Anatomie zu schreiben, das für den älteren Studenten, aber auch für den Chirurgen als Hilfsmittel zur raschen Orientierung dienen sollte. Das Buch ist 1949 erstmals erschienen und liegt demnächst in 5. Auflage vor, einer Erweiterung und Verbesserung der ersten Auflage.

Ich kann diesen kurzen Bericht über meine langjährige Tätigkeit am anatomischen Institut nicht abschließen, ohne die Arbeit meiner Mitarbeiter zu würdigen und ihnen für ihre loyale Unterstützung aufrichtig zu danken.

Bei der Übernahme des Lehrstuhles im Wintersemester 1944/45 standen mir Otto Bucher und Adolf Faller zur Seite. Faller folgte bereits 1946 einem Ruf an das Institut für Histologie in Fribourg, während Bucher auf das Sommersemester 1950 nach Lausanne übersiedelte, wo er seither dem Institut für Histologie vorsteht. Der Grundstein seines erfolgreichen und von den Studenten sehr geschätzten Lehrbuches der Histologie wurde hier in Zürich gelegt. Es erschien in erster Auflage im Jahre 1948.

Im Rahmen einer Hilfsaktion für die schwer notleidenden holländischen Universitäten übernahm die Universität Zürich Ende 1944 das Patronat über die Landesuniversität Leiden. Im Sommer 1945 wurden Dozenten zu einem Erholungsurlaub in unser Land eingeladen. Unter ihnen befand sich auch J.Dankmeijer, der im Herbst 1946 die durch den Weggang von Faller verwaiste Stelle als Gastdozent übernahm. 1947 wurde er durch W. Nauta abgelöst, der bis Ende des Sommersemesters 1951 als sehr beliebter Dozent am Institut wirkte. Sein Hauptbetätigungsfeld war die Neuroanatomie. Er siedelte in die USA über, wo er seine Hirnforschungen mit großem Erfolg weiterführte. Heute bekleidet er eine Professur am Institute of Technology in Boston (Mass.).

In den auf den Weggang von Nauta folgenden Jahren wurden K. Theiler, R. Schenk und J. Rickenbacher meine engsten Mitarbeiter. Robert Schenk 
verließ das Institut 1956 und wirkt heute als Professor der systematischen Anatomie in Bern. Er wurde durch St. Kubik ersetzt, der 1957 zusammen mit anderen Landsleuten von Budapest nach Zürich kam.

Ewald R. Weibel, Ordinarius und Direktor des anatomischen Institutes in Bern, begann seine Laufbahn in Zürich, wo er 1955 eine Assistentenstelle übernahm. Mit einem Stipendium der Schweizerischen Akademie der Medizinischen Wissenschaften konnte er sich in New Haven und New York weiterbilden. Nach seiner Rückkehr wurde ihm die Leitung der von ihm eingerichteten Abteilung für Elektronenmikroskopie übertragen, die er mit großem Erfolg führte. Nach seinem Weggang im Jahre 1966 wurde diese von Gonzague Kistler übernommen, der sie weiter ausbaute, so daß das Institut heute über eine sehr gut eingerichtete Forschungsabteilung verfügt.

\section{Literatur}

1. Bargmann, W.: Wilhelm von Möllendorff (6.12.1887-10.2.1944). Z. Zellforsch. Mikr. Anat. 33, 167-186, 1944.

2. Baumgartner, C.: Der Anatom Walther Felix 1860-1930 (Zch. med. gesch. Abhandl., Neue Reihe Nr. 132), Juris, Zürich 1979.

3. Bautzmann, H.: Walther Vogt zum Gedächtnis. Naturwiss. 29, 345-347, 1941.

4. Bluntschli, H.: Hedwig Frey (1877-1938). Viertelj.schr. Nat. forsch. Ges. Zürich 84, 341-349, 1939.

5. Fick, R.: Zur Erinnerung an H.v.Meyer und Bemerkungen über Knochen- und Gelenkformung. Verh. Anat. Ges. (Frankfurt a/M.), Erg. H. Anat. Anz. 66, 3-14, 1928.

6. Françillon, M. R.: Hedwig Frey. Anat. Anz. 88, 182-189, 1939.

7. Fürbringer, M.: Heidelberger Professoren aus dem 19.Jahrhundert. Festschrift der Universität Heidelberg zur Centenarfeier ihrer Erneuerung. 2. Band, S. 1 ff., 1903.

8. Gagliardi, E., H.Nabholz und J.Strohl: Die Universität Zürich 1833-1933 und ihre Vorläufer. Festschrift zur Jahrhundertfeier. Verlag der Erziehungsdirektion, Zürich 1938.

9. Goerttler, K.: Walther Vogt zum Gedächtnis. Anat. Anz. 92, 184-196, 1941/42.

10. His, Wilh.: Zum Gedächtnis an Karl Ludwig. Königl. Sächs. Ges. d. Wiss. math.-phys. Classe 1895.

10a. His, Wilh.: Karl Ludwig und Karl Thiersch. Allg. Zeitung Nr.164 und 165, 1895 (München).

10b. Huber, A.: Theodor Billroth in Zürich 1860-67. Diss. Zürich 1924.

11. Koch, F.: Der Anatom Georg Hermann von Meyer 1815-1892. (Zch. med. gesch. Abhandl., Neue Reihe Nr. 131), Juris, Zürich 1979.

12. Koller, H.: Das Anatomische Institut der Universität Zürich in seiner geschichtlichen Entwicklung. Diss. Zürich 1926.

13. Merkel, F.: Jacob Henle, ein deutsches Gelehrtenleben. Braunschweig 1891. 
14. Meyenburg, H.v.: Prof. Dr. Walther Felix. Sep. Druck NZZ, März 1930.

15. Meyer-Ahrens, K.: Die Geschichte des medizinischen Unterrichts in Zürich. Denkschr. med.-chir. Ges. Kt.Zürich zur Feier des 50. Stiftungstages. Zürich 1860.

16. Meyer-Ahrens, K.: Die Ärztefamilie von Muralt. Schw. Zschr. f. Heilkunde I, 268, 436, und II, 25, 1862.

17. Meyer von Knonau, G.: Der Kanton Zürich, 2.Band. St. Gallen und Bern 1834, 1846.

18. Schlaginhaufen, O.: Georg Ruge 1852-1919. Sep. Druck NZZ, 3. Febr. 1919.

19. Schlaginhaufen, O.: Walter Hermann Felix. Anat. Anz. 72, 355-360, 1931.

20. Spemann, H.: Walther Vogt zum Gedächtnis. Roux' Arch. Entw. Mech. 141, 1-14, 1941.

21. Schultze, O.: Gedächtnisrede auf Philipp Stöhr. Verh. Phys.-Med. Ges. Würzburg XLII, $1-12,1912$.

22. Stöhr, Ph.: Gedächtnisrede auf Albert von Kölliker. Verh. Phys.-Med. Ges. Würzburg XXXVIII, 277-298, 1906.

23. Töndury, G.: Wilhelm von Möllendorff, 6.12.1887-10.2.1944. Viertelj. schr. Nat. forsch. Ges. Zürich 89, 141-145, 1944.

24. Töndury, G.: Zur Transplantation des dorsalen Urmundlippenmaterials in die präsumptive Medullaranlage. Viertelj. schr. Nat. forsch. Ges. Zürich 89, 35-44, 1944.

24a. Töndury, G.: Der Versuch einer Integration des anatomisch-physiologischen Unterrichtes an der Universität Zürich. In: W. Bargmann, Aus der Werkstatt des Anatomen. Georg Thieme, Stuttgart 1965.

25. Waldeyer, W.: Georg Hermann von Meyer. Dtsch. Med. Wschr. 34, 1915.

26. Zürcher Spitalgeschichte, Band 1. Herausgegeben vom Regierungsrat des Kantons Zürich 1951.

\section{Summary}

This article gives a short survey on the beginnings of teaching anatomy in Zürich in the 18th century, and its development in the years which followed the inauguration of the University in 1833. A detailed description follows for the years 1920 to 1970 with a particular characterisation of the professors who held the chair of anatomy during this time. Furthermore the teaching methods are discussed, and in this connection it is referred to the integration of the lectures in anatomy, physiology and biochemistry, which are given together in Zürich since 1956, following a determined, carefully thought out plan. In this way, both specialities which in 1856 were separated, became re-united.

Prof.Dr.med. Dr.h.c. Gian Töndury

Stettbachstraße 11

CH-8702 Zollikon/Zürich 